\title{
Ultrathin Sub-Terahertz Half-Wave Plate with High Conversion Efficiency based on Zigzag Metasurface
}

\author{
Alexia Moreno-Peñarrubia, Sergei A. Kuznetsov, Member, IEEE, and Miguel Beruete
}

\begin{abstract}
In this paper, an ultrathin transmissive half-wave plate based on a bi-layered zigzag metasurface operating at the lower-frequency edge of the THz spectrum is numerically and experimentally studied. The half-wave plate thickness is only $100 \mu \mathrm{m}$, less than $\lambda / 20$ at the operation frequency and achieves an amplitude transmission efficiency over $90 \%$ and a cross polarization discrimination around $30 \mathrm{~dB}$ within a fractional bandwidth near $9 \%$. A detailed analysis of the device robustness with respect to layer misalignments is carried out by designing and fabricating two additional devices with the maximum possible shift between layers along both transverse directions. The results show that the device is extremely robust relative to a misalignment along $x$ and exhibits a frequency shift with misalignments along $y$, while keeping in all cases an excellent performance as a half-wave plate. The paper ends with a final study to ascertain a physical mechanism that explains the robustness of the device in regard to misalignments. These results complement and extend the reach of metasurfaces in the emerging terahertz band.
\end{abstract}

Index Terms - circular polarization, electromagnetic radiation, half-wave plate, metasurfaces, terahertz radiation, polarizer.

\section{INTRODUCTION}

The unique electromagnetic response of materials in the terahertz (THz) band $-0.1-10 \mathrm{THz}-$ as well as the noticeable progress in the techniques of $\mathrm{THz}$ generation and detection over the last decades has led to improving conventional and developing novel THz-related technologies. [1]. Advancement in $\mathrm{THz}$ instrumentation enabled elaborating numerous planar quasi-optical $\mathrm{THz}$ components such as flat lenses [2] or reflectarrays [3], tending towards more compact and lightweight devices.

Manuscript received XXX; revised XXXX; accepted XXXXXX. Date of publication XXXXXX; date of current version XXXXXXX. This work was supported by the Spanish Ministerio de Ciencia, Innovación y Universidades project RTI2018-094475-B-I00 (MCIU/AEI/FEDER, UE). The work of A. Moreno-Peñarrubia was supported by the UPNA through the FPI Ph.D. Grant Program. The work of S. A. Kuznetsov was supported by the Russian Science Foundation, Project \#19-12-00103. (Corresponding author: Miguel Beruete.)

A. Moreno-Peñarrubia is with the Antennas Group-TERALAB, Universidad Pública de Navarra, 31006 Pamplona, Spain (e-mail: alexia.moreno@unavarra.es).

S. A. Kuznetsov is with the Physics Department, Novosibirsk State University, 630090 Novosibirsk, Russia, and with the Rzhanov Institute of semiconductor Physics, 630090 Novosibirsk, Russia (e-mail: sakuznetsov@nsu.ru)

M. Beruete is with the Antennas Group-TERALAB, Universidad Pública de Navarra, 31006 Pamplona, Spain, and also with the Institute of Smart Cities (ISC), Universidad Pública de Navarra, 31006 Pamplona, Spain (e-mail: miguel.beruete@unavarra.es). Color versions of one or more of the figures in this communication are available online at $\mathrm{http}$ ://ieeexplore.ieee.org.Digital Object Identifier XXXXXXXXXXXX
Metasurfaces, the two-dimensional (2D) equivalent of metamaterials, are ideal candidates for high-efficiency quasi-optical $\mathrm{THz}$ components due to their capability of modifying locally the electromagnetic response within a subwavelength range [4], [5], facilitating device miniaturization in a fully planar geometry compatible with conventiona manufacturing techniques. These characteristics make metasurfaces very attractive for advanced wave manipulation devices, such as compact waveplates.

Waveplates are the key components in photonics and modern optics, as they allow for a full control of the electromagnetic wave polarization of high relevance in some applications, e.g. communication systems [6]. Quarter-wave plates (QWP) are intended to convert a linear polarization to a circular one [7], [8]. Recently, a transmissive selfcomplementary zigzag (ZZ) QWP metasurface working at microwaves was reported in [9]. This design exhibits interesting characteristics such as a high conversion efficiency, an experimental relative 3-dBaxial-ratio (AR) bandwidth (BW) of 53\% and high stability for oblique incidence.

Another element for polarization control is the half-wave plate (HWP), designed to impose a phase shift of $180^{\circ}$ between orthogonal linear polarization components at the output, having both components identical amplitude of the transmission coefficient (ideally, equal to unity). These devices find application in beam splitters for polarization-sensitive imaging applications [10] and have been revisited in the last few years by proposing designs based on anisotropic, bianisotropic and chiral metasurfaces [11]-[14]. Most of these designs are presented only in a theoretical way.

Most examples of HWP working at $\mathrm{THz}$ found in literature operate in reflection mode [15] and [16]. HWPs working in transmission mode, which is more useful in practice, and in a compact format are in general more difficult to synthesize. The reason is the impossibility of achieving $100 \%$ of efficiency in transmission with a single-layer structure, since this requires simultaneous fine-tuning between the electric and magnetic response [17]. In [18], a HWP utilizing cut-wirepairs metasurfaces with two layers working in transmission mode was demonstrated. However, the polarization conversion efficiency was below $80 \%$ and the overall thickness was relatively high, around $\lambda / 10$. In [19] a plasmonic metasurface HWP operating in sub-THz range was introduced achieving a broad band operation with an efficiency above $80 \%$. Finally, [20] and [21] study multilayer designs working in transmission with efficiencies near to $90 \%$ and thickness of $\lambda / 20$ and $\lambda / 8$, respectively, with an operation frequency around $10 \mathrm{GHz}$.

In this paper, we get inspiration from the results of the ZZ-QWP discussed in [9] and extend them to widen the technological reach of the $\mathrm{ZZ}$ geometry with the goal of working in a band of technological interest nowadays and where the manufacturing and experimental characterization is more difficult than at other bands like microwaves. With this aim in view, we propose and demonstrate a new bilayer ZZ metasurface to obtain an ultracompact HWP device operating near $150 \mathrm{GHz}$. The manufactured device achieves a conversion efficiency 
higher than $90 \%$ with a thickness of $\lambda / 20$ and a near-unity ellipticity of the output wave, ensuring quasi-perfect circular polarization conversion, improving results found in the literature both in efficiency and compactness.

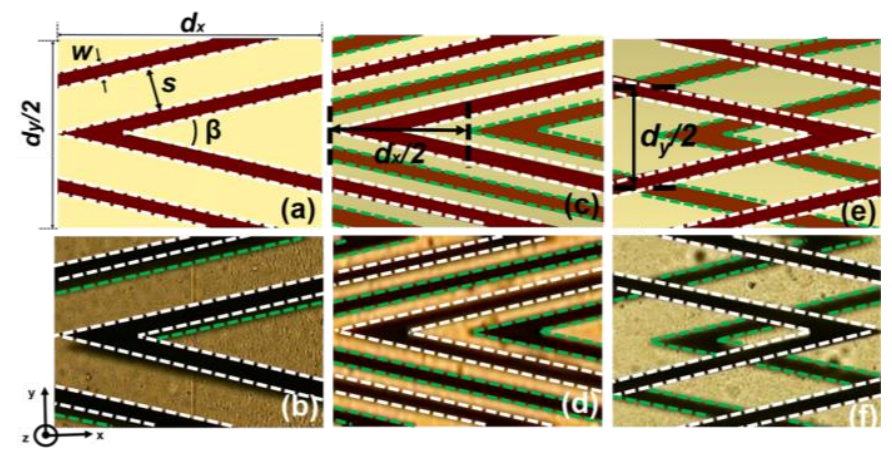

Fig. 1. Schematic representation (left) and microphotograph (right) of the ZZHWP unit cells corresponding to: (a)-(b) no-shifted, (c)-(d) $x$-shifted and (e)-(f) $y$-shifted structure. The shifted structures have maximum shift increments between layers for $x$ - and $y$-axis, $\Delta x=d_{x} / 2$ and $\Delta y=d_{y} / 2$, respectively. The green and white dashed lines have been included to help the visualization of opposite metallic layers (dark strips). The pictures of the fabricated devices were taken with a Mitutoyo Hyper MF U176-402-43 microscope with 20X zoom and using the bottom light illumination to simultaneously observe both faces.

\section{Design, SIMULATION AND EXPERIMENTAL RESUlTS}

Three different ZZ metasurface HWPs were designed and manufactured (see the unit cells in Fig. 1). All the devices are designed with the same parameters $w=20 \mu \mathrm{m}, \beta=25 \mathrm{deg}$ and $s=60 \mu \mathrm{m}$ (although manufacturing introduces some unavoidable errors) and consist of a bilayered ZZ-pattern made of aluminum strips with thickness $t_{a}=0.55 \mu \mathrm{m}$ separated by a thin flexible polypropylene (PP) film with permittivity 2.25 and thickness $t_{s}=0.1 \mathrm{~mm}$. PP was intentionally chosen as the substrate material due to its low dielectric losses $\left(\tan \delta \cong 1 \times 10^{-3}\right)$ and low dispersion in the $\mathrm{THz}$ band [22]. In all the calculations, made with the commercial simulator CST Microwave Studio ${ }^{\circledR}$, the aluminum conductivity was taken as $2.7 \times 10^{7} \mathrm{~S} / \mathrm{m}$, smaller than the DC nominal value in order to account for extra losses introduced by roughness.

In the first design, hereinafter referred to as no-shift, the ZZ-patterns in both faces are parallel, and therefore they appear overlapped in the schematic representation of Fig. 1(a). Although inspired by the QWP presented in [9], the final design must inevitably deviate from a pure self-complementary geometry due to the fact that it is a bilayer structure and it has a PP spacer, two constraints that fall out of the Babinet's theorem conditions. Therefore, a fine-tuning of the structure was mandatory to get a proper HWP operation around $150 \mathrm{GHz}$ with the widest possible frequency bandwidth. This tuning was performed using the frequency domain solver of CST. A fine tetrahedral mesh with 134000 cells was used with a minimum and maximum edge mesh cell lengths of $0.48 \mu \mathrm{m} \quad\left(0.000248 \lambda_{0}\right)$ and $286 \mu \mathrm{m} \quad\left(0.14 \lambda_{0}\right)$ respectively. Unit cell periodic boundary conditions were defined along $x$ - and $y$-axes and open space along $z$. The excitation was done with two Floquet ports using circular polarization modes. After this fine-tuning, the final parameters of the $\mathrm{ZZ}$ were obtained: width $w=20 \mu \mathrm{m}$, gap $s=60 \mu \mathrm{m}$, angle $\beta=25^{\circ}$. The size of the unit cell was $d_{x}=360 \mu \mathrm{m}$ along $x$-axis and $d_{y}=260 \mu \mathrm{m}$ along $y$-axis.

Having parallel ZZ-patterns is the best configuration for an optimal HWP performance, as shown below. An ideal HWP switches completely the polarization state of a circularly polarized wave at the input to the orthogonal polarization with maximum power transfer at the output. In the simulation results of Fig. 2(a), we get a maximum magnitude of the cross-polar transmission coefficient $\left(T_{X P}\right)$ around $-0.7 \mathrm{~dB}$ whereas the magnitude of the co-polar transmission coefficient $\left(T_{C P}\right)$ falls below $-30 \mathrm{~dB}$, meaning an excellent polarization conversion at the design frequency. To evaluate more accurately the performance, we use herein the cross-polar discrimination (XPD), a parameter defined as the ratio between the cross- and co-polar transmission coefficient magnitudes:

$$
X P D=20 \log \left|\frac{T_{X P}}{T_{C P}}\right|=20 \log \left|\frac{T_{L R(R L)}}{T_{R R(L L)}}\right|
$$

where the subscripts $R$ and $L$ refer to right-handed or left-handed circular polarization respectively. With this definition, it is clear that the design criterion for an HWP is to maximize the parameter XPD as much as possible. In the simulation curve of Fig. 2(b) a peak value of near $30 \mathrm{~dB}$ is obtained at the design frequency with a fractional bandwidth of $8 \%$, where the bandwidth is defined as the frequency span where the XPD is above $10 \mathrm{~dB}$. To corroborate experimentally the designed ZZ-HWP performance, three prototypes with non-shifted and shifted metasurface layers were fabricated by a photolithographic technology, see picture in Fig. 1(b). Prior to micro-patterning, the PP film was metallized from both sides via thermal deposition of aluminum in vacuum and the ZZ-pattern was created afterwards by using a contact photolithography technique, as described in [22].
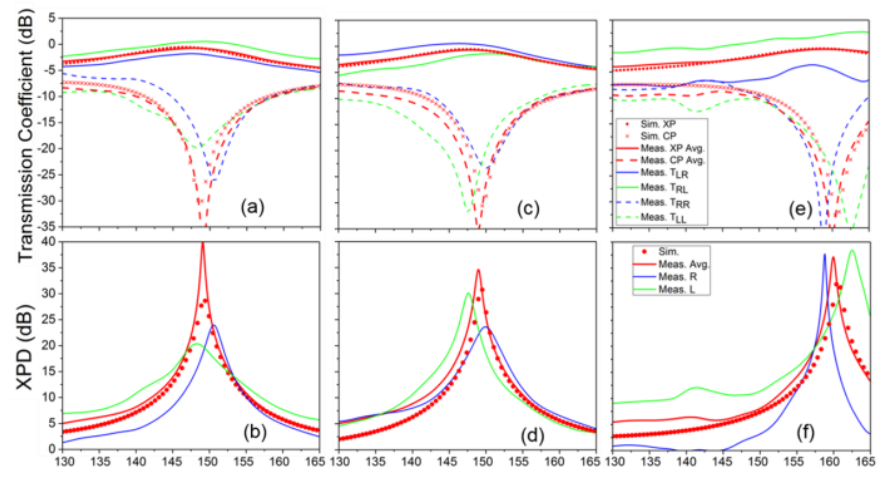

Fig. 2. Transmission coefficient magnitude for the (a) no-shift, (c) $x$-shift and (e) $y$-shift cases. XPD for the (b) no-shift, (d) $x$-shift and (f) $y$-shift cases. In the legend, Sim. stands for simulation, Meas. for measurement, XP for cross-polar, $C P$ for co-polar, $A v g$. for average, $T$ for transmission coefficient and the subscripts $R$ and $L$ for right-handed and left-handed circular polarizations.

The measurements were performed with the setup depicted schematically in Fig. 3. It consists of an $\mathrm{ABmm}{ }^{\mathrm{TM}}$ millimeter-wave vector network analyzer (VNA) operating in the D-band of the millimeter-wave spectrum that extends from 110 to $170 \mathrm{GHz}$ and equipped with a quasioptical bench. For the characterization, a vertically polarized Gaussian beam with high purity is emitted into free space by a corrugated horn antenna (TX) and a pair of elliptical mirrors collimates the beam at the sample location. The transmitted beam is then redirected by another pair of mirrors towards the receiving antenna (RX), which is also a corrugated horn. As we want to obtain the performance in a circular basis and the antennas are linearly polarized, the measurement procedure consists in doing first a calibration of the system by recording the free-space transmission with both TX and RX antenna vertically polarized. The HWP is inserted in two different configurations $\left(0^{\circ}\right.$ and $\left.90^{\circ}\right)$ and two measurements are taken at each configuration by rotating the RX antenna $0^{\circ}$ and $90^{\circ}$ as well, in order to record the vertical and horizontal components, respectively. In this way, we obtain four linear transmission coefficients $\left(T_{u u}, T_{u v}, T_{v u}, T_{v v}\right)$ in complex domain, that cover all linear co- and cross-polar scenarios, as represented in the insets. 
The transmission matrix in a circular basis can be straightforwardly obtained from them by simply applying a basis transformation:

$$
\begin{gathered}
C=\left(\begin{array}{ll}
T_{R R} & T_{R L} \\
T_{L R} & T_{L L}
\end{array}\right)= \\
\frac{1}{2}\left(\begin{array}{cc}
T_{u u}+T_{v v}+j\left(T_{u v}-T_{v u}\right) & T_{u u}-T_{v v}-j\left(T_{u v}+T_{v u}\right) \\
T_{u u}-T_{v v}+j\left(T_{u v}+T_{v u}\right) & T_{u u}+T_{v v}-j\left(T_{u v}-T_{v u}\right)
\end{array}\right)
\end{gathered}
$$

An ideal HWP should have $T_{R R}=T_{L L}=0$ and $T_{R L}=T_{L R}=1$ that correspond to $T_{u u}=-T_{v v}=0$ and $T_{u v}=T_{v u}=0$. However, in practice these conditions are fulfilled only approximately. The matrix $C$ relates the fields at the input and output in a circular polarization basis:

$$
\left(\begin{array}{c}
E_{R}^{o} \\
E_{L}^{o}
\end{array}\right)=C\left(\begin{array}{c}
E_{R}^{i} \\
E_{L}^{i}
\end{array}\right) .
$$

where $E$ is the electric field and the superscript $i$ and $o$ stand for input and output, respectively. Thus, to get the most complete characterization possible, below we present the results by assuming analytically a right-handed and left-handed circularly polarized wave at the input. This means that for the co-polar transmission coefficient, we will consider both $T_{R R}$ and $T_{L L}$, obtained in complex domain and from them we will calculate an average of them, expressing the resulting magnitude in $\mathrm{dB}$ and denoted as $C P A v g$. in the plots. Likewise, for the cross-polar transmission coefficient we will consider both $T_{R L}$ and $T_{L R}$ (also in complex domain) and from them we will calculate an average value, expressing the resulting magnitude in $\mathrm{dB}$ and denoted as XP Avg. in the plots. As a side comment, note that the calculation of an average is important because, as it will be seen, there are some curves that reach values slightly above $0 \mathrm{~dB}$ (obviously, unphysical).

Moreover, an obvious discrepancy in $T_{R L}$ and $T_{L R}$ transmission coefficients occurs, being more remarkable in the $y$-shift case, for instance, where a difference of almost $5 \mathrm{~dB}$ is obtained. This can be attributed to experimental errors such as system position misalignments (the rotation of the RX antenna and the sample were done manually) and noise. Also, as $T_{R L}$ and $T_{L R}$ are calculated from $T_{u u}$, $T_{u v}, T_{v u}, T_{u v}$, (complex coefficients), small phase errors in these linear coefficients due to thermal deviations in the VNA can have a big impact in the results. Normally, the measurements are fast and this is not a big problem. However, in the present case for a fixed sample position, two different measurements are performed, co- and crosspolar by rotating the RX antenna. The calibration is done with antennas in copolar position. The rotation of the RX antenna for the crosspolar characterization requires some manipulation time, which might be critical as it can introduce some unavoidable thermal deviation of the VNA in this extremely sensitive measurement.

Furthermore, the quasioptical bench plate can have different effect on vertical and horizontal polarization, which is usually negligible thanks to the focusing mirrors that guide the Gaussian beam. However, it could happen that the sample expands the beam so that it effectively "sees" the ground plane below, leading to a different response under vertical or horizontal polarization. All these factors can add together leading to a discrepancy between $T_{R L}$ and $T_{L R}$. Nevertheless, a systematic series of measurements were performed until we found that by averaging, the results were consistent with simulations. As a double check, we measured the cross-polar transmission isolation (in linear polarization, i.e. with TX and RX antennas in orthogonal positions) in absence of DUT and found that it is reasonably low (below $25 \mathrm{~dB}$ ). Likewise we characterized the magnitude and phase response with antennas parallel and found negligible variation in the measured spectrum (not shown).

Following the method described, we obtain the rest of the curves shown in Fig. 2(a). Both cross-polar components $T_{L R}$ (solid blue) and
$T_{R L}$ (solid green) reach a peak at the operation frequency and their average value $X P$ (solid red) shows outstanding agreement with the simulation. Regarding the copolar components $T_{R R}$ (dashed blue) and $T_{L L}$ (dashed green), both exhibit a clear dip around the operation frequency and the average value $C P$ (dashed red) almost coincides with the simulated curve. This excellent performance is corroborated in the $X P D$ results of Fig. 2(b). Again, there are two cases to consider: assuming right-handed (blue) or left-handed (green) polarization at the input. The $X P D$ is calculated as appears in the rightmost term of (1) in either case.

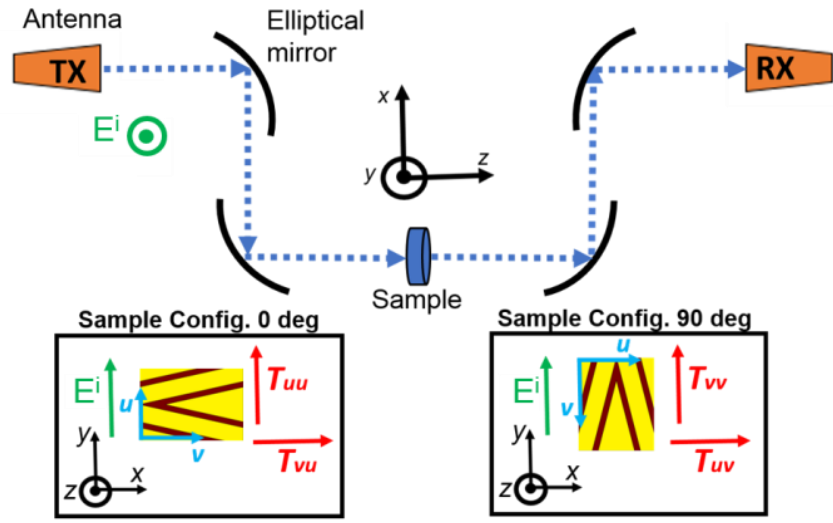

Fig. 3. Schematic of the experimental setup. The insets show two ZZ-HWP configurations. For both configurations, the linear transmission coefficients are obtained with both TX and RX antennas parallel to $y$ and with the TX antenna parallel to $y$ and $\mathrm{RX}$ antenna parallel to $x$.

An obvious peak near the operation frequency is obtained in both left and right cases, manifesting excellent concordance with the simulation curve, with a maximum value that almost reaches $40 \mathrm{~dB}$. This value, higher than theoretical one, is obtained by using XP and $\mathrm{CP}$ average magnitude values (drawn in red solid and dashed lines respectively in the leftmost graphs of Fig. 2), but each of the measurement values is lower, around $30 \mathrm{~dB}$, so it could be a fortunate effect of the selected averaging method. Therefore, we take as a realistic performance an $X P D$ of around $30 \mathrm{~dB}$. Thus, from the previous analysis we can affirm that the no-shift design has an excellent behavior, with very low experimental insertion loss $(<1 \mathrm{~dB})$ meaning a circular polarization conversion efficiency around $90 \%$, high XPD ( $-30 \mathrm{~dB})$ and large fractional bandwidth $(\sim 8.3 \%)$. To offer a more detailed view, the main performance parameters are summarized in Table I.

\section{ROBUSTNESS ANALYSIS WITH MISALIGNED DEVICES}

Even though the previous results are encouraging, we know that the manufacturing process can introduce some inevitable deviations, amongst which the alignment between layers is especially relevant, as the microphotograph of Fig. 1(b) reveals. As demonstrated in the previous section, those small imperfections were not important and did not deter the ZZ-HWP behavior from the simulation results. Nevertheless, as a proper layer alignment might be time-consuming in a massive production, we evaluate now the robustness of the device against misalignment. We test two additional designs considering the worst case of maximal shifting along $x$ - and $y$-directions, shown in Fig. 1(c), (d) and Fig. 1(e), (f), and hereinafter called as $x$-shift and $y$-shift, respectively. Concentrating first on the $x$-shift design, one can notice the robustness of the ZZ-HWP with respect to misalignment errors in this dimension: both the simulation and experimental results are nearly coincident with those of the no-shift prototype studied above. As before, the average measurements almost overlap with the simulation curves, as shown in Fig. 2(c). Similarly, the average $X P D$ value reaches 
a high peak at the operation frequency with a maximum value of approximately $30 \mathrm{~dB}$, low insertion loss $(<1 \mathrm{~dB})$ and a fractional bandwidth near $8 \%$. A detailed analysis to explain the robustness with respect to a shift in the $x$-direction is carried out in the next section.

In contrast, when the misalignment is applied in the $y$-direction, the response of the ZZ-HWP is severely affected, see Fig. 2(e). The main spectral change observed is a frequency upshift of the optimal polarization conversion, that now takes place near $160 \mathrm{GHz}$, i.e. a deviation of $6.4 \%$ with respect to the design frequency. Nevertheless, the device still works well as a HWP, with experimental insertion losses below $1 \mathrm{~dB}$, a maximum XPD larger than $35 \mathrm{~dB}$ and a fractional bandwidth of $14 \%$. The reason for this change is studied in the next Section. The most representative values for every model are displayed in Table I. A comparison of the present structure with other recent works of HWP based on metasurfaces is presented in Table II. As observed there, the ZZ-HWP has an excellent XPD value as well as a broad operation bandwidth, a remarkable result for a device operating in transmission. In addition, the structure is extremely compact.

\section{PHYSICAL OPERATION: FIELD ANALYSIS}

Now, we analyze in detail the reason why the structure has a strong robustness with respect to a shift in the $x$-direction but it is more sensitive to a shift along $y$. We start the numerical analysis by performing two parameter sweeps: varying $\Delta x$ and $\Delta y$ from zero (fully aligned) to the maximum misalignment value, $d_{x} / 2$ or $d_{y} / 2$, respectively, assuming circular polarization.

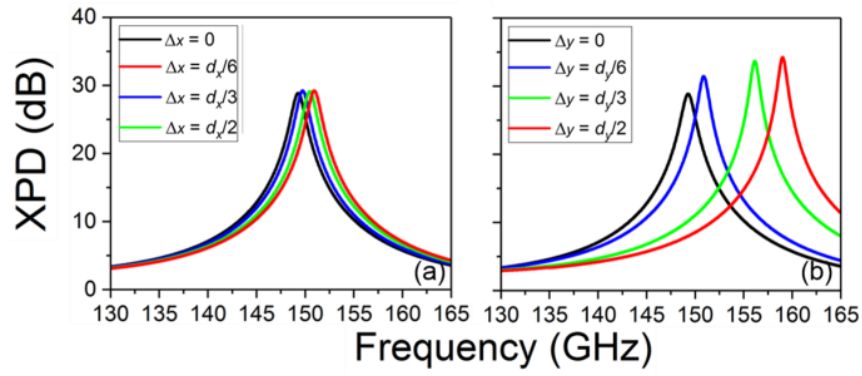

Fig. 4. Simulated $X P D$ for several misalignments: (a) $\Delta x$ and (b) $\Delta y$.

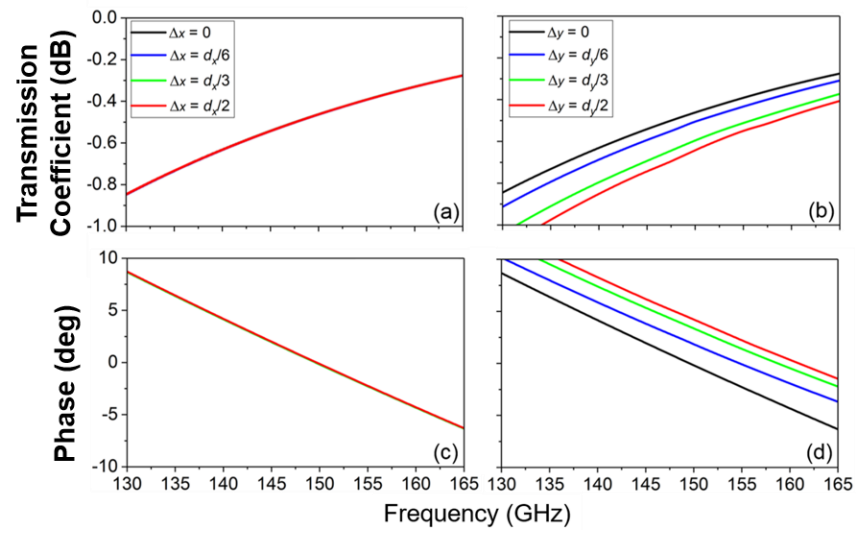

Fig. 5. Simulated vertical polarization transmission coefficient for various (a), (c) $\Delta x$ and (b), (d) $\Delta y$ cases. Top and bottom panels show magnitude and phase, respectively.

From the results, presented in Fig. 4, we can deduce that the HWP operation frequency increases in both cases, but the effect is considerably stronger for $\Delta y$. To understand this dissimilar behavior, we carry out a field analysis in linear components, considering both linear polarizations. Thus, under vertically polarized excitation, the device is largely transparent with a transmission coefficient magnitude close to $0 \mathrm{~dB}$ in the entire frequency span and a phase near $0^{\circ}$ at the operation frequency, see Fig. 5. As observed there, the response is almost insensitive to $\Delta x$ and presents some negligible dependence on $\Delta y$, with a variation in magnitude of less than $0.2 \mathrm{~dB}$ and in phase of less than $5^{\circ}$ at the operation frequency.

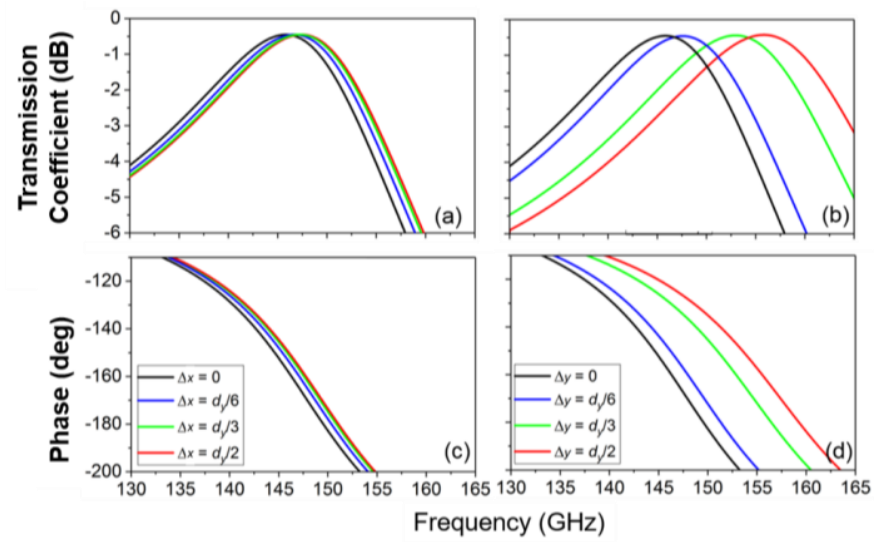

Fig. 6. Simulated horizontal polarization transmission coefficient for various (a), (c) $\Delta x$ and (b), (d) $\Delta y$ cases. Top and bottom panels show magnitude and phase, respectively.

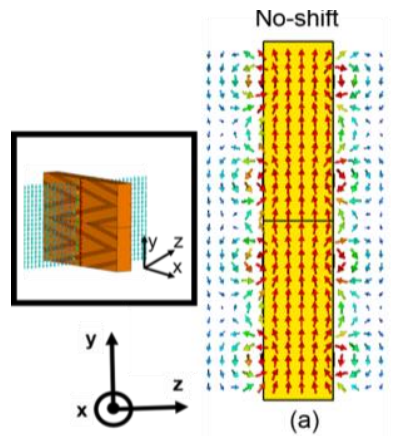

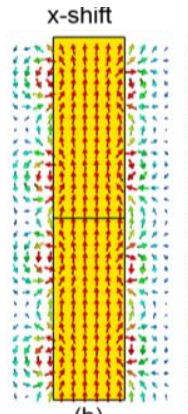

(b)

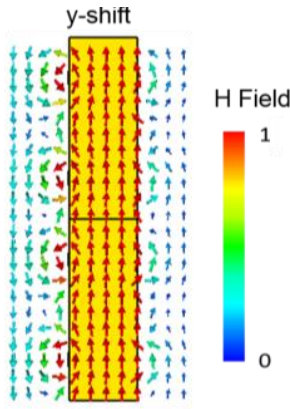

(c)
Fig. 7. Cross-sectional view ( $y-z$ plane as shown in the leftmost inset) of the instantaneous magnetic field (represented as arrows) for (a) no-shift, (b) $x$-shift and (c) $y$-shift designs at the respective operation frequency. (a)

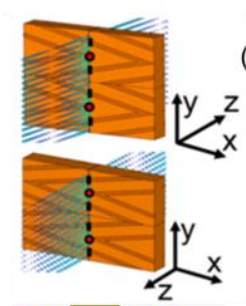

(b)

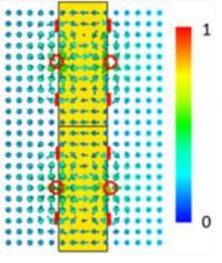

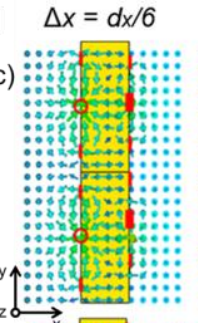

(d)

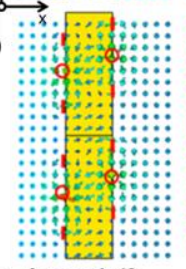

$\Delta y=d y / 6$
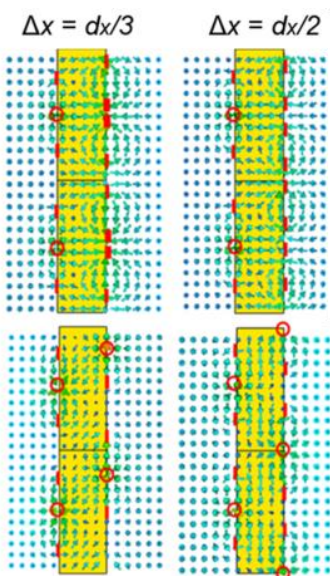

$\Delta y=d y / 2$
Fig. 8. (a) Front (top) and back (bottom) views showing the cross-section (noshift case) where the electric field is evaluated. The cross-sectional view ( $y-z$ plane) of the instantaneous electric field (represented as arrows) at the maximum $X P D$ frequency for (b) no-shift, (c) different $\Delta x$ and (d) different $\Delta y$ cases. ZZ vertices and the strip cross-sections have been highlighted with red circles and straight segments to aid with the interpretation, following the scheme shown in (a).

In contrast, the response with horizontal polarization is also almost insensitive to $\Delta x$ but demonstrates a marked variation with $\Delta y$, as can 
be inferred from the simulated transmission coefficient in Fig. 6. In all cases, there is a peak in the magnitude that happens near but not exactly at the point where the phase is $180 \mathrm{deg}$ and whose location depends strongly on $\Delta y$. Overall, from this initial analysis, it is clear that the horizontal polarization component is the main responsible for the performance of the misaligned ZZ-HWP. Thus, hereafter we fix the attention to this case.

As observed in Fig. 8(c), when $\Delta x$ is varied, the electric field lines are nearly identical to the no-shift case. This suggests that this modification still allows an electric coupling between layers in a similar way as the ideal case. As a consequence, the operation frequency remains almost unmodified, probably due to a similar value of the capacitance between layers. However, when the misalignment is applied along $y$, the electric coupling is strongly affected and, in fact, the electric field in the dielectric spacer differs substantially from the initial no-shift case. From the plots in Fig. 8(d), it can be concluded that the electric coupling and hence the capacitance between layers diminishes. This explains the upshift of the operation frequency.

\section{CONCLUSIONS}

To sum up, in this paper we have analyzed in detail the performance of the ultrathin transmissive HWP based on a bi-layered $\mathrm{ZZ}$ metasurface designed for operation at $150 \mathrm{GHz}$. Having the thickness of $\lambda / 20$, the fabricated device revealed excellent agreement with the numerical simulations, reaching a maximum transmission coefficient magnitude of $-0.78 \mathrm{~dB}$ (i.e. the polarization conversion efficiency is over $90 \%$ ), and an $X P D \sim 30 \mathrm{~dB}$, with a fractional bandwidth near $9 \%$.

The robustness of the device with respect to possible misalignments between layers introduced in the manufacturing process has been analyzed in detail. From the analysis performed, it has been clarified that the response is extremely robust with regards to a shift along the $x$ direction whereas it varies more considerably with a shift along the $y$ direction. It is worth noting that even in the worst case, the performance of the device as a HWP still remains excellent although shifted in frequency.

A subsequent study has clarified that the response is very stable for any misalignment under linear vertical polarization excitation, but it is very dependent to misalignments along $y$ under linear horizontally polarized excitation. We have ascertained that this is due to the strong electrical coupling between layers, whose field distribution has a strong dependence on a shift along $y$.

The results of this work extend and complement other approaches to develop polarization conversion devices, in a compact realization and with excellent performance, such as low insertion losses and a relatively broad bandwidth. Moreover, the analysis presented here is general and could be extended to other frequency ranges such as microwaves or infrared by properly rescaling the geometry parameters of the metasurface.

TABLE I

ZZ-HWP PARAMETERS FOR MEAS. AVG.

\begin{tabular}{ccccc}
\hline \hline Model & $X P D_{\max }(d B) \mathrm{a}$ & $f_{X P D}(\mathrm{GHz}) \mathrm{ad}$ & $T x(d B)^{*}$ & $B W(\%)^{* *}$ \\
\hline No-Shift Sim. & 28.87 & 149.25 & -0.742 & 8.09 \\
No-Shift Meas. & 39.14 & 149.11 & -0.785 & 8.98 \\
x-Shift sim & 31.11 & 149.35 & -0.709 & 8.27 \\
x-Shift Meas. & 34.75 & 149.08 & -0.668 & 9.20 \\
y-Shift Sim. & 34.30 & 159.02 & -0.650 & 9.16 \\
y-Shift Meas. & 37.03 & 159.04 & -0.751 & 9.72 \\
\hline \hline
\end{tabular}

a $f_{X P D}$ frequency at XPD maximum.

ad $X P D_{\max }$ maximum XPD value.

* $T x$ corresponds to the transmission amplitude value at the frequency where $X P D$ is maximum.

** $B W$ is the frequency bandwidth considering $X P D$ values higher than $10 \mathrm{~dB}$.
TABLE II

COMPARISON WITH OTHER HWP DEVICES

\begin{tabular}{ccccccc}
\hline \hline REF & Mode & Layers & $\begin{array}{c}X P D_{\max } \\
(d B)\end{array}$ & $\begin{array}{c}f_{X P D} \\
(T H z)\end{array}$ & $\begin{array}{c}B W \\
(\%)\end{array}$ & $\begin{array}{c}\text { Thickness } \\
(\lambda)\end{array}$ \\
\hline$[15]$ & $\mathrm{RX}$ & 2 & $\sim 20$ & 2 & 32 & $\sim 1 / 12$ \\
{$[16]$} & $\mathrm{RX}$ & 2 & $\sim 25$ & 95 & 42 & $\sim 1 / 5$ \\
{$[17]$} & $\mathrm{TX}$ & 3 & 13.97 & 0.6 & 2 & $\sim 1 / 5$ \\
{$[18]$} & $\mathrm{TX}$ & 2 & 18.06 & 0.5 & 0.6 & $\sim 1 / 14$ \\
{$[19]$} & $\mathrm{TX}$ & 1 & 26.74 & 0.14 & 10.7 & $\sim 1 / 4$ \\
{$[20]$} & $\mathrm{TX}$ & 2 & 13.06 & 0.0101 & 4.9 & $\sim 1 / 20$ \\
{$[21]$} & $\mathrm{TX}$ & 3 & 25.2 & 0.0105 & 7.6 & $\sim 1 / 8$ \\
Present & $\mathrm{TX}$ & 2 & 39.14 & 0.149 & 8.98 & $\sim 1 / 20$ \\
Work & $\mathrm{T}$ & & & & & \\
\hline \hline
\end{tabular}

\section{REFERENCES}

[1] Y. S. Lee, Principles of Terahertz Science and Technology, 1st ed. Boston, MA: Springer US, 2009.

[2] C.-C. Chang, D. Headland, D. Abbott, W. Withayachumnankul, and H.-T. Chen, "Demonstration of a highly efficient terahertz flat lens employing tri-layer metasurfaces," Opt. Lett., vol. 42, no. 9, p. 1867, May 2017.

[3] T. Niu et al., "Experimental demonstration of reflectarray antennas at terahertz frequencies," Opt. Express, vol. 21, no. 3, p. 2875, Feb. 2013.

[4] H.-T. Chen, A. J. Taylor, and N. Yu, "A review of metasurfaces: physics and applications," Reports Prog. Phys., vol. 79, no. 7, p. 076401, Jul. 2016.

[5] S. B. Glybovski, S. A. Tretyakov, P. A. Belov, Y. S. Kivshar, and C. R. Simovski, "Metasurfaces: From microwaves to visible," Phys. Rep., vol. 634, pp. 1-72, May 2016.

[6] M. S. Kong et al., "Terahertz radiation using log-spiral-based lowtemperature-grown InGaAs photoconductive antenna pumped by mode-locked Yb-doped fiber laser," Opt. Express, vol. 24, no. 7, p. 7037, Apr. 2016.

[7] D. Wang, Y. Gu, Y. Gong, C.-W. Qiu, and M. Hong, “An ultrathin terahertz quarter-wave plate using planar babinet-inverted metasurface," Opt. Express, vol. 23, no. 9, p. 11114, May 2015.

[8] B. Yang, W.-M. Ye, X.-D. Yuan, Z.-H. Zhu, and C. Zeng, "Design of ultrathin plasmonic quarter-wave plate based on period coupling," Opt. Lett., vol. 38, no. 5, p. 679, Mar. 2013.

[9] J. D. Baena, S. B. Glybovski, J. P. del Risco, A. P. Slobozhanyuk, and P. A. Belov, "Broadband and Thin Linear-to-Circular Polarizers Based on Self-Complementary Zigzag Metasurfaces," IEEE Trans. Antennas Propag., vol. 65, no. 8, pp. 4124-4133, Aug. 2017.

[10] J. S. Tyo, D. L. Goldstein, D. B. Chenault, and J. A. Shaw, "Review of passive imaging polarimetry for remote sensing applications," Appl. Opt., vol. 45, no. 22, p. 5453, Aug. 2006.

[11] V. S. Asadchy, A. Díaz-Rubio, and S. A. Tretyakov, "Bianisotropic metasurfaces: physics and applications," Nanophotonics, vol. 7, no. 6, pp. 1069-1094, Jun. 2018.

[12] J. E. Roy and L. Shafai, "Reciprocal circular-polarization-selective surface," IEEE Antennas Propag. Mag., vol. 38, no. 6, pp. 18-33, Dec. 1996.

[13] X. Wu, Y. Meng, L. Wang, J. Tian, S. Dai, and W. Wen, "Anisotropic metasurface with near-unity circular polarization conversion," Appl. Phys. Lett., vol. 108, no. 18, p. 183502, May 2016.

[14] P. E. Sieber and D. H. Werner, "Infrared broadband quarter-wave and half-wave plates synthesized from anisotropic Bézier metasurfaces," Opt. Express, vol. 22, no. 26, p. 32371, Dec. 2014. L. Peng, X.-F. Li, X. Jiang, and S. Li, "A Novel THz Half-Wave Polarization Converter for Cross-Polarization Conversions of Both Linear and Circular Polarizations and Polarization Conversion Ratio Regulating by Graphene," J. Light. Technol., vol. 36, no. 19, pp. 4250-4258, Oct. 2018.

[16] X. Zhang, D. Kong, Y. Yuan, S. Mei, and G. Wang, "Broadband and dispersion-free reflective silver metasurfaces as half-wave plate and vortex-beam generator," Opt. Commun., vol. 465, no. February, p. $125561,2020$.

[17] M. Jia et al., "Efficient manipulations of circularly polarized terahertz waves with transmissive metasurfaces," Light Sci. Appl., vol. 8, no. 1, p. 16, Dec. 2019.

[18] Y. Nakata, Y. Taira, T. Nakanishi, and F. Miyamaru, "Freestanding 
transparent terahertz half-wave plate using subwavelength cut-wire pairs," Opt. Express, vol. 25, no. 3, p. 2107, Feb. 2017.

X.-P. Dong, J.-R. Cheng, F. Fan, S.-T. Xu, X.-H. Wang, and S.-J Chang, "Wideband sub-THz half-wave plate using 3D-printed lowindex metagratings with superwavelength lattice," Opt. Express, vol. 27, no. 1, p. 202, Jan. 2019.

[20] M. R. Akram, M. Q. Mehmood, X. Bai, R. Jin, M. Premaratne, and W. Zhu, "High Efficiency Ultrathin Transmissive Metasurfaces," Adv. Opt. Mater., vol. 7, no. 11, p. 1801628, Jun. 2019.

[21] W. Luo, S. Sun, H.-X. Xu, Q. He, and L. Zhou, "Transmissive Ultrathin Pancharatnam-Berry Metasurfaces with nearly $100 \%$ Efficiency," Phys. Rev. Appl., vol. 7, no. 4, p. 044033, Apr. 2017. M. Navarro-Cia, S. A. Kuznetsov, M. Aznabet, M. Beruete, F. Falcone, and M. S. Ayza, "Route for Bulk Millimeter Wave and Terahertz Metamaterial Design," IEEE J. Quantum Electron., vol. 47, no. 3, pp. 375-385, Mar. 2011.

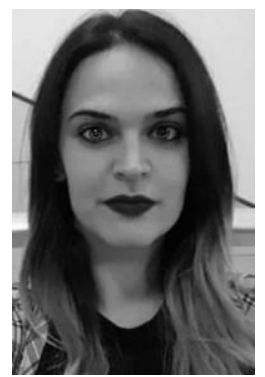

Alexia Moreno-Peñarrubia received the M.Sc. degree in telecommunication engineering from the Polythecnic University of Valencia, Spain, in 2014. She was part of the Radiation Electromagnetic Group in the iTEAM reasearch institute, Polytechnic City of Innovation, Valencia, as junior researcher, where she developed a work related to antenna arrays and Butler matrix feeding networks in the microwave range, which was awarded with the Young Scientist Award 2015 by the URSI committe. From 2015 to 2017 she worked at TTI Norte Company, in Cantabria, Spain and at Tryo Aerospace in Barcelona, Spain as RF Antenna Engineer. Currently, she is pursuing the Ph.D. degree under the supervision of Prof. M. Beruete in the Antennas Group, Public University of Navarre, Pamplona, Spain. Her current research interests include metasurfaces, polarization converter structures, lenses and holography in the $\mathrm{THz}$ range.

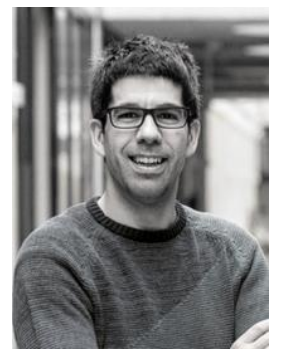

Miguel Beruete was born in Pamplona, Spain, in 1978. He received the M.Sc. and Ph.D. degrees in telecommunication engineering from the Public University of Navarre (UPNA), Pamplona, in 2002 and 2006, respectively.

From 2002 to 2007, he was a PreDoctoral Researcher, under FPI fellowship, with the Electrical and Electronic Engineering Department, UPNA. In 2005, he was a Visiting Researcher with the University of Seville, Seville, Spain, as a part of his Ph.D. research. From 2007 to 2009, he was with the Electronics Department, Technological Center CEMITEC, Noáin, Spain, where he was involved in the development of high frequency communication devices. In 2009, he joined TERALAB, UPNA, as a Post-Doctoral Researcher on the Consolider EMET Engineering Metamaterials Project. From 2012 to 2016, he was a Ramon y Cajal Fellow Researcher with UPNA, where he became an UPNA Distinguished Researcher. Since 2017, he has been an Associate Professor with the Antennas Group-TERALAB, UPNA, where he supervises several Ph.D. and M.Sc. theses and leads the TERALAB Laboratory. He has authored more than 130 indexed articles, 5 book chapters, nearly 250 conference communications (several invited). He holds three patents. His current research interests include terahertz sensing and communication technology, including metamaterials, plasmonics, extraordinary transmission structures, leaky-wave antennas, nanoantennas, and in general quasi-optical devices.

Dr. Beruete was a recipient of the Ph.D. Prize from UPNA for the Best Doctoral Thesis from 2006 to 2007, three CST University Publication Awards for the best international journal publication using CST in 2005, 2012, and 2016, the XII Talgo Award of Technological Innovation in 2011, and several awards of international conferences. He is a reviewer for more than 50 international journals. 